\title{
SKADS: Scope and Overview of Activities and Results
}

\author{
A. van Ardenne $e^{1,2}$ \\ 1 ASTRON, Oude Hoogeveensedijk 4, 7991 PD Dwingeloo, The Netherlands \\ ${ }^{2}$ Chalmers University of Technology, SE-412 96 Gothenburg, Sweden

\begin{abstract}
An introductory overview of the SKA Design Studies is given. This includes a short history, SKADS objectives, its structure and organization, its impact and an account of the work performed of which this SKADS Conference book bears full witness.
\end{abstract}

\section{Introduction}

It was understood very early on that the very wide frequency range for the SKA is realizable only as a combination of receiving technologies e.g. with sparse arrays, dense arrays and with dishes. While sparse narrow-band phased arrays are as old as radio astronomy, the new electronically steered, widefield multi-octave sparse and dense arrays hold immense scientific potential through their flexibility and widefield multibeaming capability. However, these array concepts being new are relatively immature and technically and scientifically essentially unproven. Radio observatories, in particular in Europe, therefore became increasingly more convinced that a structured and broadly supported R\&D effort on phased arrays was necessary to advance emerging views on wide field astronomy as a key characteristic for the SKA van Ardenne \& Butcher (2003). This view was stimulated by most of the Key Science Projects mentioned in the SKA Science book Carilli \& Rawlings (2004). Earlier technical R\&D activities planned as exploratory steps of the new array concept supported the feasibility of the approach (e.g. van Ardenne \& Butcher 2003; van Ardenne et al. 2000; Hall 2004, and references therein) resulting in early technical developments for LOFAR at the end of the nineties.

As a consequence, a proposal emphasising aperture arrays (AAs) for the SKA was submitted to the European Commission FP6 Research program on behalf of the European SKA Consortium. The focus was on the dense array concept noting the need for functional integration, low cost (materials), low power and manufacturability.

"Dense" refers to the distance between neighboring antenna elements being less than half a wavelength at the maximum frequency, thereby limiting the maximum frequency for practical and cost reasons. Clearly identified in the early R\&D phase van Ardenne \& Butcher (2003); Schaubert et al. (2003) this array concept constituted a paradigm shift as it was proven to operate over a multi-octave frequency band combined with a constant rather than decreasing (with increasing frequency) aperture area. This solved a major outstanding issue in the array element selection. It also allows other elements than the socalled Vivaldi to be used because the constant area array feature is inherently based on the strong mutual coupling between the (preferred) elements rather than a "disturbance" as in common $\Omega$ radar array design.

The developments and results for low frequency arrays such as LOFAR act as pathfinders for the larger and global context of SKA. They provide important input to the Aperture Array approach, for example with respect to calibration and processing techniques. Other parallel developments explore the use of dense focal plane arrays. In Europe, activities started through the Radionet EC-FP5 program "FARADAY" followed by Radionet "PHAROS" within EC-FP6, and subsequently "APERTIF" as an upgrade program for the WSRT. Similar developments took place in the US, in Canada and for the Australian SKA pathfinder. These arrays enlarge the field of view of existing reflector telescopes as well as providing a "field enhancing" candidate technique for the SKA. See the contributions in these Proceedings and SKADS Memos ${ }^{\mathrm{a}}$ plus references therein for a recent overview of new phased arrays for radio astronomy in general.

With this as background, the key objectives of the proposed SKA Design Studies "SKADS" SKADS Consortium (2007) were to

1. Demonstrate SKA Scientific viability and readiness for frequencies below $1.4 \mathrm{GHz}$,

2. Demonstrate cost-effective engineering solutions and Technological Readiness and

3. Arrive at a costed SKA design.

Other objectives are placing SKADS into the framework of the SKA planning and engagement models and to endorse a European SKA activity, involving industries in some key areas to establish the relevant and distributed R\&D platform. Note that SKADS does not focus on monitoring and scheduling nor on data archiving, storage, distribution and user interface. While their importance is clear, the rationale was that SKADS had to focus on concept specific elements only.

Approved to start in mid-2005 as an EC supported FP6 program, SKADS was planned to last four years. It initially involved 31 institutes and industries in 8 European countries with participants in the Russian republic, South Africa, Canada and Australia. At mid-term, this focused to 26 institutes with 9

\footnotetext{
${ }^{a}$ http://www.skads-eu.org/p/memos.php
} 
European countries now including Portugal, thus enlarging the European basis.

The program received significant EC funding of $10.44 \mathrm{M} €$ supplemented by contributions from national funding in an approximately $2.5: 1$ ratio. This initial value is thus over $36 \mathrm{M} €$ in total including all effort at the level of over $80 \mathrm{FTE}^{\mathrm{b}}$ per year. As SKADS progressed, the contributions reduced for various reasons, but without affecting the major outcomes. An extension of SKADS for 6 months until the end of 2009, mainly because of slower progress with the demonstrators, was proposed and subsequently approved by the EC.

\section{Structure and Organization}

SKADS was structured to bring together the various aspects of Research \& Development necessary for optimum results as a series of studies. In the Description of Work the so-called Design Study ("DS") activities relate to:

1. Science and Astronomical Data simulations to model the sky to match with the potential SKA instrumental capabilities. Then to simulate the data and imaging pipeline (DS2)

2. System level design in close collaboration with DS2 to identify requirements for station level processing; to configure, control, connect, correlate the array stations and simulate the overall cost (DS3). DS2 and DS3 work on the specification and architectural design of the SKA. Together they amount to about $25 \%$ of the SKADS effort. In this way SKADS structure continuously maps the scientific requirements onto the technical specifications to produce an optimum design.

3. Technology developments on key issues were planned in DS4, to advance the technology maturity and to select and prove the various enabling technologies at component level. The DS4 effort amounted to about $30 \%$ of the total. It included the delivery of a Dual Polarisation All Digital array (2-PAD) as a technology demonstrator.

4. Development Demonstrators to bring the technology developments from component to subsystem level and conclude on an integrated performance. DS5 and DS6 in total amount to about $30 \%$ of SKADS. DS5 focuses on the development and test of the Electronic Multi Beam Radio Astronomy ConcEpt (EMBRACE) consisting of two dense array stations, one at ASTRON's Westerbork site of about $175 \mathrm{~m}^{2}$ and a smaller version at the Nançay Observatory of about $80 \mathrm{~m}^{2}$. DS6 focuses on the development of the Basic Element for SKA Training (BEST), a platform for early technology tests around the Medicina cylindrical array.

The remaining effort was the overall SKADS management, finances, communication and outreach through the SKADS Program Office (DS1). The assessment of work as scheduled Project Reviews such as the Quarterly reports and the MidTerm Review (DS7) and the consolidated, final deliverable i.e. the SKADS overall system design as part of a Whitepaper (DS8) involving all DS leaders and selected others.

\footnotetext{
${ }^{\mathrm{b}}$ FTE=Full Time Equivalent $=$ one year of effort by one person
}

SKADS focused on concept specific elements emphasising the station, the configuration, the network and the associated technical costs.

The six DS leaders coordinated the 23 Tasks within the Design Studies, each led by a task leader of a participating institute. Prior to submitting the SKADS proposal the distribution of responsibilities and the mapping of the participants capabilities was solved in a complex and detailed process. The participants could therefore commit to all the objectives and over 60 deliverables detailed in the SKADS Description of Work SKADS Consortium (2007).

All SKADS results (foreground knowledge) are available in the public domain. This is covered by a Consortium Agreement signed by all partners together with an Intellectual property Rights, IPR, document protecting some (background) IPR in conformity with EC regulations. See, the SKADS website ${ }^{\mathrm{c}}$ for $\Omega$ references. An annual event, the SKADS workshop, was organized to communicate progress and promote dissemination and publication of results as in the outreach plan produced as an early DS1 deliverable.

The SKADS Marie-Curie Conferences \& Training program, a related three year FP6 program was approved. This was a focused dissemination and educational effort of SKADS re sults in parallel to the SKADS R\&D program in key areas. See the SKADS Marie Curie Conferences \& Training webpages ${ }^{\mathrm{d}}$. $C$.

\section{SKA, phased arrays and key issues to investigate}

The huge potential for SKA science return by using phased arrays is due to fundamental capabilities of such an instrument. The large field of view (FoV) available for large surveys is limited only by the elemental beam pattern, by the available processing power, and by the associated cost. Maximum flexibility is provided by electronic control and numerous digital beams. It is possible to trade FoV with frequency bandwidth using constant data rates and processing capability. Using reasonable assumptions for technical performance of e.g. achievable array system temperatures, costs, and maximum frequency, estimated around $1.4 \mathrm{GHz}$; many science key experiments mostly for the HI universe are best implemented using AAs.

At the outset, the issue of the required processing power of the SKA stood out and the novelty of AAs warranted work in SKADS. The relatively low number of around 250 stations requires far less central processing power by approximately two orders of magnitude than is required by $\sim 2000$ or more dishes. This is due to central processing increasing as the square of the number of collectors and the aperture array property that FoV is only proportional to the number of independent beams.

The SKA Life Cycle Costs need to be taken into account, of which the operational and maintenance costs are important ingredients. When considering AAs various aspects of the design limit operational costs. For example, there are no mechanically moving parts, no low temperature cooling systems are required and the high usage of electronics functionality allows

\footnotetext{
c http://www.skads-eu.org

d http://mcct.skads-eu.org
} 
for graceful operational degradation while minimizing maintenance. To quantify the performance effects, adequate calibration techniques are needed to allow for the changes to the system.

Another important consideration for the SKA is the potential for upgrading. Although an upgrade policy needs to be formulated, AAs offer upgrade paths at tile level as well as at processing level. These aspects need to be taken into account in further developments although SKADS did not consider this aspect in any detail.

Can we afford AAs? Together with the scientific potential and the engineering specifications and capabilities, the issue of the affordability of AAs is equally important i.e. can we afford AAs in the SKA framework as an investment and as part of an operating instrument. Of these two major issues, SKADS considers the capital cost primarily. Relevant elements of operating cost relate to power consumption, computation costs, maintainability/reliability etc. and also to the issue of upgradability mentioned earlier.

Over the 15 years R\&D on AAs for radio astronomy, steady progress has been made on reducing the cost to an affordable level suitable for the SKA. The cost per square metre involves the complete station including receiving, processing, communication etc. based on, for example, 250 Aperture Array Stations each about $60 \mathrm{~m}$ diameter. EMBRACE in particular shows costing progress and is now in the range of a few thousand $€ / \mathrm{m}^{2}$ depending on the computing details. For the next step, during the course of the AAVP (see elsewhere in these Proceedings) this figure is expected to reduce further, making AAs very cost effective.

The AA concept is appealing to industry as it ranks high as an innovation driver. Several industries are linked to SKADS as partners in early R\&D and/or as suppliers; the most prominent of which were IBM and OMMIC. It is foreseen that the next steps involve a more structured connection to industries often as part of national interests. At the same time, this involvement must closely match the required functional performance with industrial capabilities.

Much of the SKADS efforts go a long way in trying to answer the question not only if AAs are going to perform technically but, of course, also scientifically. The science modeling and simulations in SKADS are as far as one can go without a major instrument to demonstrate the science capability e.g. wide field scanned deep polarimetric imaging performance. See also the contribution on AAVP elsewhere in these Proceedings. In SKADS the technical way forward was first to select the key, enabling technologies, to develop and project these adequately and validate the results at subsystem level (BEST) and ultimately at demonstrator level, as in 2-PAD and EMBRACE, as input to an SKA design. Over the next year, results are expected with regard to measured scanned noise performance, tracking and pointing, predicted vs. measured beam shape (e.g. as a result of electronic changes in time and frequency), beam stability, reliability, predicted vs. real power consumption, and other aspects relevant to proving the technical performance. Additionally one can make a start with calibration algorithms using for example strong sources to further enhance the scientific potential with AAs.
The emphasis of SKADS therefore is R\&D coupled with demonstrating the design and potential of wideband, constant effective area aperture arrays for the SKA. Obviously a followup program is needed as input to the first major stage of constructing the SKA. This is the Aperture Array Verification Program (AAVP) detailed out elsewhere.

Such a program is also needed to provide further design of AAs for the SKA from the frequency range, design, power, (both scientific and technical) performance and cost perspective.

\section{SKADS achievements and results}

SKADS made progress in many ways and it is only possible to give a non-exhaustive summary. See these Proceedings for a more complete overview doing better justice to all involved. Many of the published results can also be found on the SKADS webpage $^{\mathrm{e}}$.

With respect to technologies and hardware, the initial chal lenges were huge. These have been addressed mostly in DS4, DS5 and DS6 with some staggering results.

- For example, while before SKADS only the surface was scratched in the domain of electromagnetic modeling, element selection, circuit modeling, low noise receivers and integrated antennas, they have now been modeled, simulated and built. This allows the next step from technology immaturity into the development and engineering phase to be made. The techniques were applied to 2-PAD and into EMBRACE both now consisting of dual polarized large arrays rather than, as planned in SKADS_EMBRACE initially, a single polarisation only.

- Several wideband antenna realizations were tried and measured but not yet concluded upon although as for EMBRACE most experience has now been acquired with "Vivaldi" types. There is a choice of element type.

- Although the low noise LNA work could not be finished, the important conclusion to be drawn is that an uncooled system temperature below $50 \mathrm{~K}$ from $1 \mathrm{GHz}$ upward (i.e. assuming $3 \mathrm{~K}$ sky noise) using wideband receivers is attainable in the next major phase for SKA around 2014.

- Functional phase transport solutions were shown in the lab, with corresponding written reports

- Low cost signal transport are being tested in all demonstrators notably BEST and EMBRACE

- Wideband ADC solutions (few GHz sampling frequency) were reported upon in DS4 and smaller bandwidth performance was demonstrated in 2-PAD and the hundreds of tiles in EMBRACE. It has been shown that fast sampling ADCs with low power will be available for the SKA.

- The building of the demonstrators proved a major achievement in SKADS, providing an excellent platform to further AA development and show the capability of taking AAs from the labs into the field. As an essential and planned result, low cost front-end manufacturing approaches and techniques were demonstrated. Specifically this is demonstrated in a SKADS movie "The making of EMBRACE".

\footnotetext{
${ }^{\text {e }}$ http://www.skads-eu.org/p/memos.php
} 


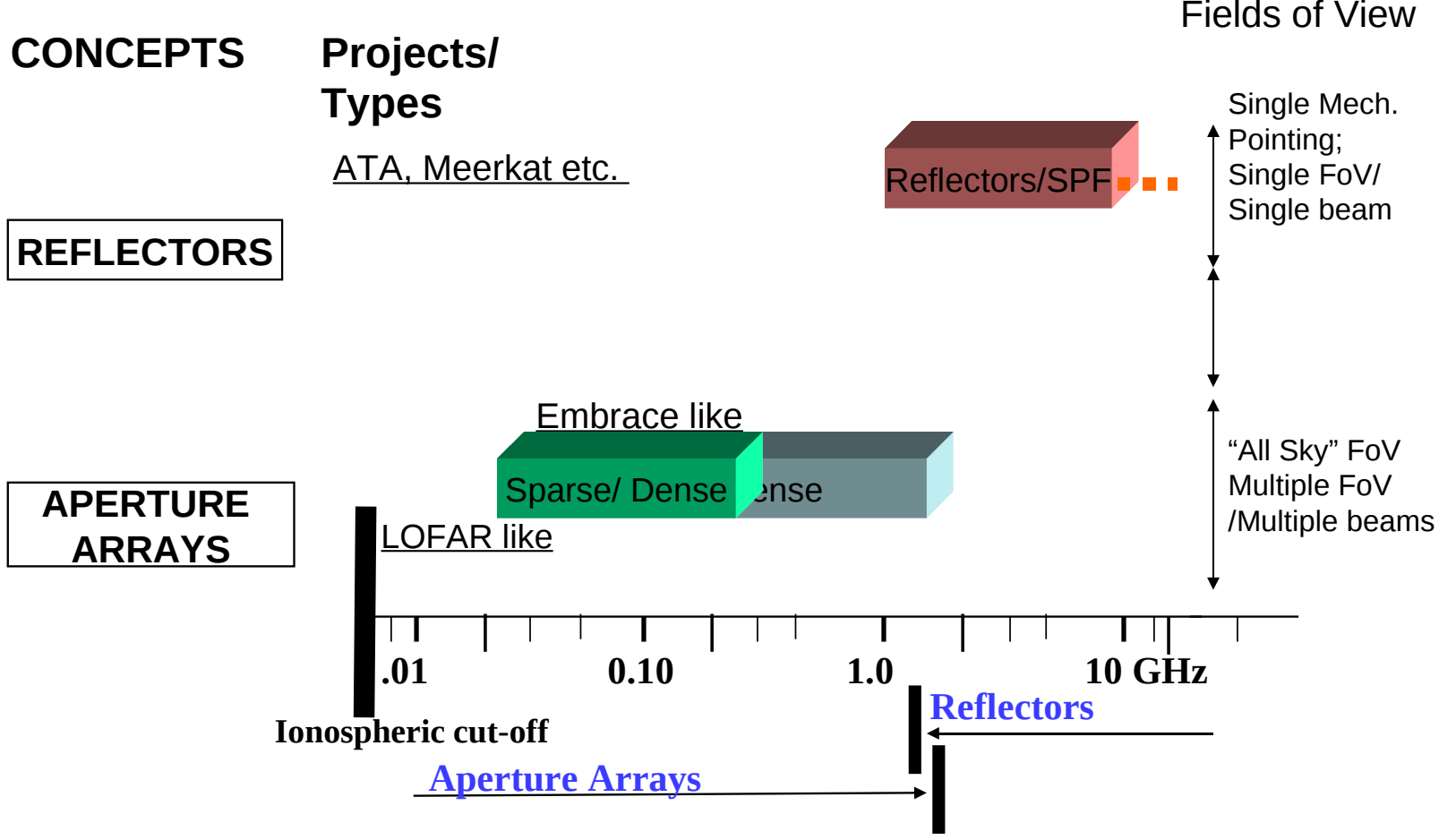

Fig. 1: The picture depicts the various collector technology conceptual approaches. In green, the range over which LOFAR and other aperture arrays are designed to operate. Great flexibility and large receiving areas are intended to be the domain of the AAs, while the dishes are designed for lowest noise at lowest cost per receiving area using single pixel feeds. This approach is costed in SKADS.

- Station level processing i.e. AA "specific" as well as SKA capable processing technology were addressed and reported. This includes beamforming technology, modeling and simulation.

A first Technology readiness level assessment was produced at the SKADS Mid Term workshop as well as in the final SKADS report, clearly indicating the areas that deserve more attention. Albeit crudely done, no major road blocks were identified that prohibit steady progress through a follow-up program (i.e. AAVP) toward AAs for the SKA.

At the System level, mostly as result of DS2 and DS3, results can be summarized as successfully modeling and simulating critical performance areas of the SKA as a science instrument. Specifically, this has been done through:

- SKA Simulated Skies for several science cases and the effect of physical conditions (e.g. configuration, number of antennas, sensitivities, atmospheres, instrumental) on the reconstructed images using Meq Trees as an astrodata reduction development tool. The simulated skies are computer simulations of the radio universe (see also SKADS Simulated Skies 2009, including the $S^{3}$ post processing toolbox) led by the University of Oxford and producing astonishing results (see e.g. Fig. 2 for an overview of the "simulated types" for public use). These activities were supported by SKA configuration studies and other simulation tools. For example, as an element of the astrodata

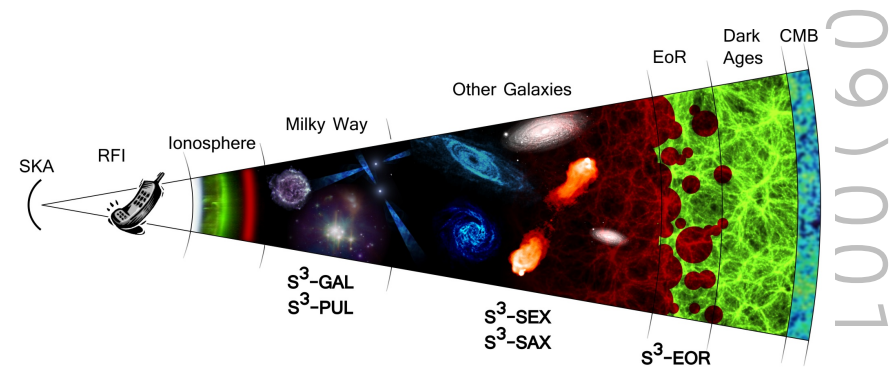

Fig. 2: This image shows a schematic representation of the different levels of simulation available from the SKADS Simulated Skies (the $S^{3}$ Tools, see Klöckner et al. 2010; Levrier et al. 2010 for more details). Image by D. Obreschkow.

simulations the OSKAR Dulwich et al. (2010) AA tool set was used to simulate the AA beam shape.

- Throughout SKADS and into the third year, a costed SKA design was produced Bolton et al. (2009) and discussed at SKA level. The status at several stages of development has been reported as SKA memos. Supporting this is a cost modeling tool that is useful for the SKA, offering a flexible, broadly usable and communicable base for costing the SKA.

- As planned, an outline of an SKA design was produced and characterized in detail for specified AA performance 
in terms of frequency, data rate, configuration, data control and network, baseline and cost etc. of an "implementable" functional and architectural design.

Longer term programs like SKADS experience issues with availability and stability of resources. Consequently, a modest level of shortcomings can be summarized in that the Demonstrators were incomplete and full array results not yet available at SKADS closure, specific decisions on technology and proof of feasibility are yet to be made which include Calibration of a dual polarisation system.

In the course of SKADS, important aspects were identified as strengths of AAs.

For example, AAs hold inherent promise of high dynamic range observing as a result of a completely unobstructed field of view and exquisite calibration capability. This of course will only work if the non-negligible beam shape changes in time and pointing can be fully calibrated out on the basis of prediction and online calibration techniques. In this respect the redundancy calibration techniques now being developed (see Wijnholds et al. 2010 and elsewhere in these Proceedings) are very promising. This is also supported by recent preliminary results using EMBRACE with only a limited number of tiles.

As the correlator capacity is increased linearly with the number of array beams, an upgrade path is constituted providing an intrinsically capable multi-beam instrument.

For example, 250 AA stations with 64 beams require the equivalent correlator power as for 2000 dishes, everything else being the same (e.g. frequency bandwidth, resolution and baseline). Putting it differently, it turns out that AAs are capable of cost mitigation of Correlation and Post processing Alexander et al. (2009).

AAs, allow the flexibility to achieve "tunability" of the number and size of stations for fixed $\mathrm{A}_{\text {eff }}$ also within stations. For the science cases in the frequency range where AAs are expected to be applied i.e. below $1.4 \mathrm{GHz}$, the emphasis is on baselines up to a few hundred kilometres. Even so, depending on the specifics of the lay-out, significant network investment cost increase at the $100 \mathrm{M} €$ level is associated with more evenly distributed (5 arms logarithmic) as compared to a more concentrated array.

\section{Conclusion and further work}

We have presented an overview of SKADS, its structure, the issues and the emphasis of the major efforts and achievements. SKADS successfully contributed to the exploration of widefield techniques for the SKA emphasising AAs and demonstrating the viability of the concept for the next SKA phase. As a fringe benefit, SKADS contributed to bring into focus the SKA as a major science project inside Europe and demonstrated Europe's key role in driving an innovative SKA.

Acknowledgements. These proceedings bear witness of all tangible results achieved in SKADS with many researchers, some of whom joined the field of Radio Astronomy through SKADS and the associated SKADS Marie Curie program. As coordinator, I am grateful to see that it is the minds, creativity and perseverance of these colleagues and their collaboration that make challenges like SKADS turn into a success. Specifically I like to thank the SKADS management team Steve Torchinsky, Andrew Faulkner, André van Es and Truus van den Brink for their undiminished support and Elena Righi-Steele as EC-FP6 program officer for her immeasurably important patience, insight and support. Last but not least all DS leaders, the SKADS Board, the SPDO and other participants and in particular the SKADS Board Chairman, Peter Wilkinson who acted as (pre)conditioner, as an enthusiastic supporter and amazingly good summariser of all meetings, and Parbhu Patel and Jan Geralt bij de Vaate who were incredibly important to the transformation of many hours and pizzas in the various versions of the DoW!

\section{References}

Alexander, P., Faulkner, A., SKA memo 108, 2009, http:// wWw. skatelescope.org/pages/page_papers.htm, see also SKADS memos (2009)

Bolton, R. et al., SKA memo 111, 2009, http://www. skatelescope.org/pages/page_papers.htm, see also SKADS memos (2009)

Carilli,C., Rawlings,S.(Eds), Science with the Square Kilometre Array, New Astronomy Reviews 48, Elsevier 2004

Dulwich, F., Mort, B.J., Salvini, S., Zarb Adami, K., Jones, M.E. "OSKAR: Simulating Digital Beamforming $\Omega$ for the SKA Aperture Array" 2010, in Proc. Wide Field Science and Technology for the SKA, Limelette, Belgium, S.A. Torchinsky et al. (eds), ASTRON, ISBN 978-90805434-5-4

Hall, P.J.(Ed.), The Square Kilometre Array; an Engineering Perspective, Springer, ISBN 1-4020-3798-8, 2005, Reprinted from Exp. Astronomy, Vol 17, nos 1-3, 2004

Klöckner, H.-R., et al., "SKA HI end2end simulation", 2010, in Proc. Wide Field Science and Technology for the SKA, Limelette, Belgium, S.A. Torchinsky et al. (eds), ASTRON, ISBN 978-90-805434-5-4

Levrier, F. et al., "Mapping the SKA Simulated Skies with the $S^{3}$-Tools," 2010, in Proc. Wide Field Science and Technology for the SKA, Limelette, Belgium, S.A. Torchinsky et al. (eds), ASTRON, ISBN 978-90805434-5-4

Schaubert, D.H., Boryssenko, A.O., van Ardenne, A., Bij de Vaate, J.G., Craeye, C., The Square Kilometre Array (SKA) Antenna, IEEE Phased Array Systems and technology Symposium, pp 351-358, Boston, USA, October 2003

SKADS memos, http://www.skads-eu.org/p/memos. php containing many published SKADS results

SKADS Consortium, SKADS Description of Work, downloadable from http://www.skads-eu.org/p/contracts. php

On Sky simulations: http://s-cubed.physics.ox.ac.uk, see also SKADS memos (2009)

van Ardenne, A., Smolders, A.B., and Hampson, G.A., Active Adaptive Array Antennas for Radio Astronomy; results of the R\&D program toward the SKA, Proc. SPIE Vol.4015, pp 420-433, Munich, March 2000

van Ardenne, A., and Butcher, H.R.(Eds), The Aperture Array approach for the SKA, SKA Design concept white paper, 
SKA Design Concept memo 14 www.skatelecope.org/ pages/page_memos.htm, May 2003

Wijnholds S. J., Tol S. van der, Nijboer R. and Veen A. J. van der, Calibration challenges in future radio telescopes, SPM (IEEE signal processing magazine), January 2010 